\title{
Decreasing Missed Opportunities for HIV Testing in Primary Care through Enhanced Utilization of the Electronic Medical Record
}

\author{
Ann K. Avery*, Michelle Del Toro and Douglaus Einstadter \\ Case Western Reserve University, Cleveland, Ohio, USA
}

\begin{abstract}
Objectives: We sought to decrease "missed opportunities" for HIV testing by implementing an electronic medical record based intervention designed to increase HIV testing among previously untested men and women ages 18-64 seeking primary medical care in an urban publicly-funded health care system.

Methods: In July 2010, we implemented an electronic medical record based reminder to alert providers to the absence of an HIV test among all patients' ages 13-64 years old. We compared the rate of missed opportunities for HIV testing among primary care patients seen during the two and a half years before the intervention with that of patients seen during the two years after the intervention was begun. A "missed opportunity" was defined as the failure of a previously untested patient to obtain HIV testing despite having made one or more primary care office visits during a specified time period.

Results: After the implementation of HIV testing reminders, first-time HIV testing increased significantly for both men and women 18-64 years old, resulting in a significant reduction in "missed opportunities." The intervention was equally effective across different racial and ethnic groups. An increase in new HIV diagnoses after the intervention was observed, consistent with an increase in the number of individuals in the population who received testing.
\end{abstract}

Conclusions: An electronic medical record-based reminder can significantly increase HIV testing among men and women ages 18-64 who are seeking primary care services.

Keywords: HIV testing reminders; Electronic medical record-based reminder; HIV diagnoses

\section{Background}

On July 13, 2010 the White House released the National HIV/AIDS Strategy for the United States. Within that strategy there is a clear call to increase the efficiencies that allow for increased testing, identification of people living with HIV, and linking them into care [1]. The Centers for Disease Control and Prevention (CDC) estimate that 1.1 million people are living with HIV in the United States, nearly three-fourths of who are men (Centers for Disease Control and Prevention, 2008). Ohio is certainly not immune to this epidemic and as of 2011, there were 17,109 Ohioans known to be living with HIV infection, with men making up 79 percent of affected individuals [2]. The Greater Cleveland area is one of the state's highest prevalence areas with most HIV cases residing within the city limits. The Cleveland Department of Public Health reports an HIV/AIDS rate of 770.9/100,000 among all Clevelanders and 1193.5/100,000 among male Clevelanders [3]. HIV cases are concentrated in areas with high rates of poverty.

Since 2006, the CDC has recommended HIV testing at least once during their lifetime for all individuals between age 13 and 64 [4]. Prior to 2006 , testing was routinely recommended only for pregnant women, individuals at high risk or living in areas with prevalence greater than $1 \%$, or for patients seeking services for sexually transmitted infections $[5,6]$. Testing by previous guidelines also limited the number of patients tested by first requiring HIV risk assessment, a known barrier to HIV testing [7]. The revised HIV testing recommendations have received widespread support with most primary care professional organizations providing policy or consensus statements encouraging their implementation [8,9]. Widespread support for HIV testing guidelines has facilitated revision of most state laws to allow for more "routine" HIV testing $[10,11]$. Routine HIV screening leads to increased and earlier diagnosis which improves outcomes for those diagnosed, and has a public health impact through reduced transmission of the disease $[12,13]$.

Screening for other diseases including diabetes, heart disease, cervical, colorectal, and breast cancer is widely accepted because early diagnosis and treatment leads to improved long-term health and longevity. Despite their association with documented improvements in health outcomes, other widely accepted screening guidelines have less than optimal utilization $[14,15]$.

Men, as well as minority populations, are noted to have lower utilization of preventive health screenings, which poses a significant concern when the burden of a disease is predominantly among these same populations [16-19]. Consequently, any encounter with the health care system, particularly for underserved populations, should be viewed as an opportunity to offer preventive health education and age- or gender-recommended screenings. In a meta-analysis of interventions to increase immunizations and cancer screening services, rates of services were most likely to improve when supported through organizational changes including support from the electronic medical record (EMR) as well as staffing changes in organizational work flow [20,21]. We examined whether HIV test recommendations implemented through the EMR would improve HIV testing among previously untested patients seeking primary care services in our health system.

*Corresponding author: Ann K. Avery, Case Western Reserve University, Cleveland, Ohio, USA, E-mail: aavery@metrohealth.org

Received June 04, 2012; Accepted August 22, 2012; Published August 26, 2012

Citation: Avery AK, Toro MD, Einstadter D (2012) Decreasing Missed Opportunities for HIV Testing in Primary Care through Enhanced Utilization of the Electronic Medical Record. J AIDS Clinic Res S4:006. doi:10.4172/2155-6113.S4-006

Copyright: ( $) 2012$ Avery AK, et al. This is an open-access article distributed unde the terms of the Creative Commons Attribution License, which permits unrestricted use, distribution, and reproduction in any medium, provided the original author and source are credited. 


\section{Methods}

We conducted a before-after study of patients seen in primary care at a safety-net hospital system in Cleveland, Ohio. The Metro Health System (MHS) is a publicly funded academic teaching hospital in Cleveland, Ohio and the primary provider of indigent health care to the community. MHS is comprised of a major medical center, a rehabilitation hospital, a long-term care/skilled nursing center, an outpatient surgery center, and a network of satellite community-based health care centers (CHC) located in areas of high medical need. The Metro Health system is also the primary provider of Ryan Whitefunded HIV medical care, serving over 1200 patients living with HIV in 2011.

MHS supports a fully integrated electronic medical record (EMR) system (EPIC), which has been used for all outpatient encounters since 1999. One feature of the EMR is a health maintenance list that can be used for general preventive health activity (e.g., tetanus vaccination) as well as specialty practice (e.g., regular hemoglobin A1C monitoring for diabetic patients) reminders. Use of the health maintenance feature has been encouraged by an institution-supported Quality Improvement (QI) program which provides regular reports on selected quality indicators included on the health maintenance list.

Between July and December 2009, we held small group educational discussions with primary care providers at each site about the benefits of routine HIV testing for patients 18-64 years of age, barriers to its implementation, a review of the sites current HIV testing and resources for patients newly diagnosed with HIV. During the time period of the educational sessions, Ohio HIV testing law also changed to allow for more routine HIV testing [22]. Providers were informed of these changes during sessions. Providers supported the concept of increased testing but felt that lack of time limited their ability to broadly implement it, particularly the time needed to determine if a patient had previously been tested; providers supported adding HIV testing to the health maintenance reminder list.

In July 2010, "HIV testing once" was added to the health maintenance list for all individuals ages 13 to 64 . The reminder automatically identified prior testing (since 1999) within MHS and provided the date completed with a link to allow viewing of the test result.

We extracted encounter level data from the EMR for all patients 18-64 years old with one or more office visits at any one of 7 selected primary care practice sites during January 2008 through June 2012. We excluded encounters for patients 13-17 years old because of unique issues in testing among adolescents. We also excluded encounters for patients known to have HIV, as indicated by a diagnosis on their problem list.

The encounter data were grouped into calendar quarters and the primary outcome, the proportion of previously untested individuals with one or more visits during the quarter who received testing during that quarter, was determined.

For our primary analysis, we grouped the data into two time periods: 1) the time period preceding any intervention; and 2) the time period after the EMR health maintenance HIV testing recommendations were initiated. The mean proportion of previously untested patients who received testing in each time period was compared using a chi square test. Additional analyses examined the cumulative proportion of patients who had received HIV testing. Because the population was relatively stable during the study period, we did not adjust the results for differences in patient age or demographics. Additionally, since

\begin{tabular}{|c|c|c|c|}
\hline Characteristic & Overall & Period 1* & Period 2* \\
\hline Number of patients & 82,706 & 58,115 & 58,071 \\
\hline Number of visits & 425,657 & 224,325 & 201,332 \\
\hline$\%$ Female & 60.7 & 63.3 & 62.1 \\
\hline Mean Age, years (std) & $41.7(13.5)$ & $41.0(12.9)$ & $41.8(13.1)$ \\
\hline \multicolumn{4}{|l|}{ Race, \% } \\
\hline White & 29.8 & 29.7 & 29.6 \\
\hline African American & 49.5 & 49.6 & 53.1 \\
\hline Hispanic & 8.0 & 9.7 & 7.9 \\
\hline Other & 10.0 & 9.7 & 6.1 \\
\hline Unknown & 2.7 & 1.2 & 3.3 \\
\hline \multicolumn{4}{|l|}{ Insurance, \% } \\
\hline Commercial & 22.0 & 23.6 & 22.4 \\
\hline Medicare & 11.6 & 13.9 & 11.4 \\
\hline Medicaid & 30.2 & 30.0 & 31.8 \\
\hline Uninsured & 35.2 & 32.5 & 34.4 \\
\hline \multicolumn{4}{|l|}{ Primary Language, \% } \\
\hline English & 77.6 & 75.2 & 85.3 \\
\hline Spanish & 5.2 & 5.5 & 5.7 \\
\hline Other / Unknown & 17.2 & 19.3 & 9.0 \\
\hline
\end{tabular}

* Period 1 = Jan 2008 - June 2010; Period 2 = July 2010 - June 2012

Table 1: Demographics of Primary Care Population Age 18 - 64 years.

our sample size is large, even minor differences will be found to be statistically significant; the clinical meaningfulness of the results was our primary focus.

Statistical analyses were performed using SPSS 20.0 (Chicago, IL) and SAS version 9.2 (SAS Institute, Cary, NC). The study involved no direct contact with patients and all information was collected retrospectively with a waiver of informed consent. We did not evaluate individual provider performance and providers voluntarily participated in the intervention. The study was approved by the MHS IRB Protocol \#007-0590 and \#10-366.

\section{Results}

Between January 2008 and December 2011, 82,706 individual patients made a total of 425,627 outpatient visits $(284,591$ female and 141,066 male) to the 7 selected primary care clinics. The characteristics of patients overall and stratified by the two reporting periods are shown in Table 1 . The mean age was 42 years and $61 \%$ were women. Half the patients were African American and over one-third were uninsured. The majority of patients spoke English as their primary language. The demographics of the patients did not differ meaningfully between the two study time periods.

Overall there was a statistically and clinically significant increase in the proportion of patients receiving first time HIV testing after the implementation of the HIV testing reminder. Before the reminder was in use, an average of 4.3 percent of eligible patients received a first HIV test during any calendar quarter. After the EMR reminder was implemented, the average proportion of previously untested patients who received a first HIV test increased nearly four-fold to an average of $17.3 \%, \mathrm{p}<0.001$ (Figure 1). This increase in testing rate was similar for both men and women (Figure 2). Grouping the data by the entire pre- 

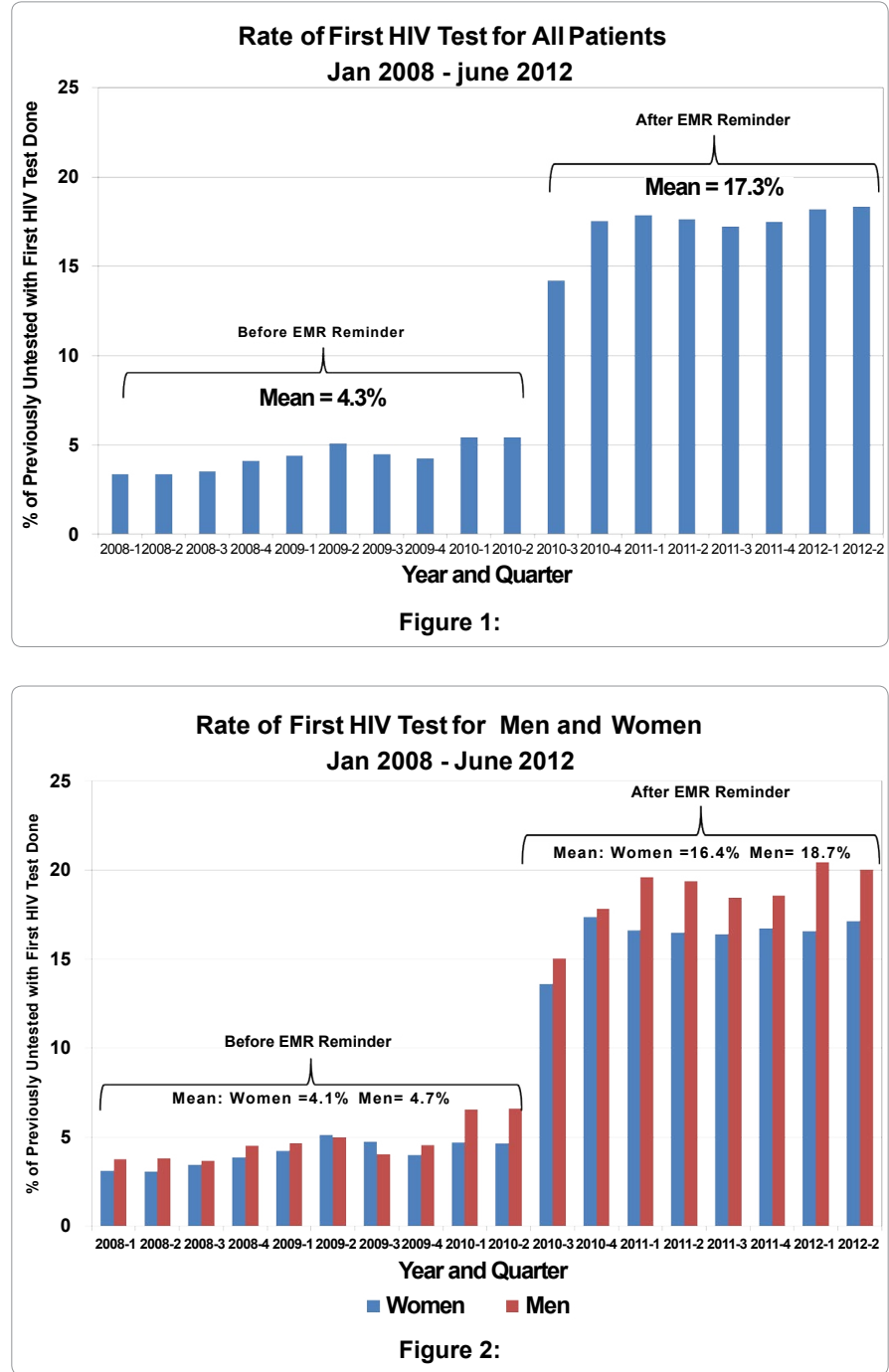

and post-intervention periods, the proportion of previously untested patients who received a first HIV test increased from 11.7 to 39.7 percent $(p<0.001)$; the increase was similar for both men and women. The intervention also was associated with a significant increase in the cumulative proportion of patients with at least one HIV test done, with the total percent of patients with HIV testing increasing from $25 \%$ to $56 \%$ for men and 40.5 to $66 \%$ for women (Figure 3).

Importantly, the rates of positive HIV tests remained stable at $0.2 \%$ among outpatients (0.6-0.8 for males and 0.1 for females) resulting in more new diagnoses in 2010 and 2011 compared to 2008 and 2009. No significant difference was noted in the rates of positive HIV tests between racial and ethnic groups between time periods. Of those newly diagnosed with HIV, greater than $80 \%$ had not been tested previously, supporting the focus of the intervention.

\section{Discussion}

The addition of an electronic record reminder was associated with a dramatic improvement in the implementation of the updated HIV testing recommendations in primary care practices. To our knowledge, this is the first description of such an intervention addressing the gap between policy and practice in HIV testing through the EMR.

We believe that replication of this intervention would be successful elsewhere. However, we must acknowledge that our providers were already accustomed to incorporate reminders from the health maintenance list and this should be taken into account in efforts to replicate the intervention. Identification of new cases among patients without identified risk factors reinforced the benefit of routine testing to providers. Additionally, no providers voiced concerns about additional time needed for testing due to the intervention.

The initial disparity in HIV testing between men and women is alarming given the overwhelming majority of cases occurring in men, especially minority men [19]. With men making fewer doctor visits each year compared to women [23], it is essential to utilize each opportunity to encourage preventive health screenings. This intervention notably addressed this disparity with dramatic decreases of missed opportunities among all adult men. It is worth noting that prenatal testing accounted for at least half of the outpatient testing in 2008 and 2009 and likely accounted for differential rates between genders of having "ever been tested." The routine approach to HIV testing in prenatal care has been extremely successful and could serve as a model for all providers of primary care.

Providers supported enhancements to the EMR and workflow changes, and should also be factored into the success of this project. It is important to note that prevalence of positive tests was maintained when testing increased; however, our intervention focused on increased testing among those never tested and this effect may not be seen if repeat testing were more common. In order to sustain an effective screening initiative, new cases need to be identified. We feel our strategy is in line with the NHAS to increase the number of individuals aware of their status. Strategies to increase first-time testing will improve the cost effectiveness of the screening tool $[14,24]$.

The evaluation has its limitations in that we did not collect data from either providers or patients on the reasons for not testing. Reasons for not testing have been well described previously [7] and these issues were addressed in the educational sessions provided but not reviewed for this analysis.

\section{Conclusion}

Routine HIV testing within medical settings provides an opportunity to test all who access the health care system without the need for the patient to acknowledge risk. It has been previously noted that patients with HIV had numerous interactions with the health care

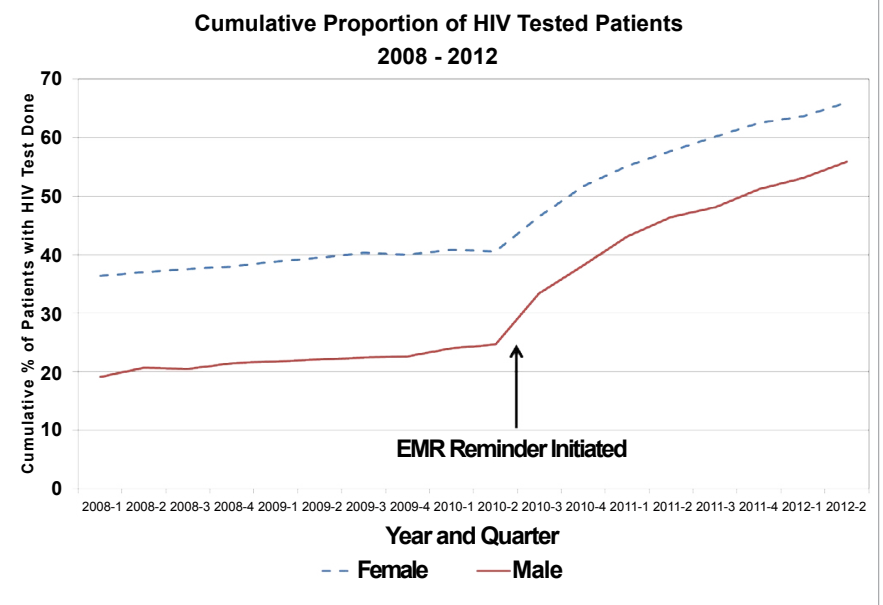

Figure 3: 
Citation: Avery AK, Toro MD, Einstadter D (2012) Decreasing Missed Opportunities for HIV Testing in Primary Care through Enhanced Utilization of the Electronic Medical Record. J AIDS Clinic Res S4:006. doi:10.4172/2155-6113.S4-006

Page 4 of 4

system prior to their diagnosis [25]. At a population level, testing all patients decreases the stigma associated with being testing for HIV, as well as allowing for earlier diagnosis and behavior change for those who test positive [26]. Additionally, for the individual who tests positive within a health care system, a holistic approach to care with enhanced supportive resources is more often available than at a free standing counseling and testing site.

Implementation of an EMR-based reminder to incorporate HIV testing into routine medical care effectively increased HIV testing to those not previously tested and should be considered by other sites that use EMRs.

\section{Acknowledgements}

This paper was funded in part by the AIDS Funding Collaborative in Cleveland, Ohio and the grant support from Jonathon Karn (Al036219).

\section{References}

1. National HIV/AIDS Strategy for the United States.

2. Ohio Department of Health 2012 Diagnoses of HIV and/or AIDS Reported in Ohio.

3. Cleveland Department of Public Health (2011) Cleveland (ONLY) HIVIAIDS Prevalence Report.

4. Branson BM, Handsfield HH, Lampe MA, Janssen RS, Taylor AW et al. (2006) Revised recommendations for HIV testing of adults, adolescents, and pregnant women in health-care settings. MMWR Recomm Rep 55: 1-17.

5. Anderson TJ, Atkins D, Cirac CB, Bayer R, Palomo FKB, et al. (2001) Revised guidelines for HIV counseling, testing, and referral. MMWR Recomm Rep 50: 1-58.

6. Recommendations for HIV testing services for inpatients and outpatients in acute-care hospital settings. MMWR 42: 157-158.

7. Burke RC, Sepkowitz KA, Bernstein KT, Karpati AM, Myers JE, et al. (2007) Why don't physicians test for HIV? A review of the US literature. AIDS 21: 16171624.

8. Qaseem A, Snow V, Shekelle P, Hopkins R Jr, Owens DK, et al. (2009) Screening for HIV in health care settings: a guidance statement from the American College of Physicians and HIV Medicine Association. Ann Intern Med 150: $125-131$.

9. ACOG Committee Opinion (2008) Routine human immunodeficiency virus screening. 112: 401-403.

10. Mahajan AP, Stemple L, Shapiro MF, King JB, Cunningham WE (2009) Consistency of state statutes with the Centers for Disease Control and Prevention HIV testing recommendations for health care settings. Ann Intern Med 150: 263-269.
11. State HIV Testing Laws. 2012 Compendium of State HIV Testing Laws.

12. Walensky RP, Freedberg KA, Weinstein MC, Paltiel AD (2007) Costeffectiveness of HIV testing and treatment in the United States. Clin Infect Dis. Dec 45: S248-S254.

13. Marks G, Gardner LI, Craw J, Crepaz N (2010) Entry and retention in medica care among HIV-diagnosed persons: a meta-analysis. AIDS 24: 2665-2678.

14. Henley SJ, King JB, German RR, Richardson LC, Plescia M (2010) Surveillance of screening-detected cancers (colon and rectum, breast, and cervix) - United States, 2004-2006. MMWR Surveill Summ 59: 1-25.

15. Berry DA, Cronin KA, Plevritis SK, Fryback DG, Clarke L, et al. (2005) Effect of screening and adjuvant therapy on mortality from breast cancer. N Engl J Med 353: 1784-1792.

16. Lees KA, Wortley PM, Coughlin SS (2005) Comparison of racial/ethnic disparities in adult immunization and cancer screening. Am J Prev Med 29 : 404-411.

17. Viera AJ, Thorpe JM, Garrett JM (2006) Effects of sex, age, and visits on receipt of preventive healthcare services: a secondary analysis of national data. BMC Health Serv Res 6: 15.

18. Qi V, Phillips SP, Hopman WM (2006) Determinants of a healthy lifestyle and use of preventive screening in Canada. BMC Public Health 6: 275.

19. (2011) Disparities in diagnoses of HIV infection between blacks/African Americans and other racial/ethnic populations--37 states, 2005-2008. MMWR Morb Mortal Wkly Rep 60: 93-98.

20. Friedberg MW, Coltin KL, Safran DG, Dresser M, Zaslavsky AM, et al. (2009) Associations between structural capabilities of primary care practices and performance on selected quality measures. Ann Intern Med 151: 456-463.

21. Stone EG, Morton SC, Hulscher ME, Maglione MA, Roth EA, et al. (2002) Interventions that increase use of adult immunization and cancer screening services: a meta-analysis. Ann Intern Med 136: 641-651.

22. http://codes.ohio.gov/orc/3701.242

23. Middleton K, Hing E, Xu J (2007) National Hospital Ambulatory Medical Care Survey: 2005 outpatient department summary. Adv Data 389: 1-34.

24. Paltiel AD, Weinstein MC, Kimmel AD, Seage GR 3rd, Losina E, et al. (2005) Expanded screening for HIV in the United States--an analysis of costeffectiveness. N Engl J Med 352: 586-595.

25. Liddicoat RV, Horton NJ, Urban R, Maier E, Christiansen D, et al. (2004) Assessing missed opportunities for HIV testing in medical settings. J Gen Intern Med 19: 349-356.

26. Beckwith CG, Flanigan TP, del Rio C, Simmons E, Wing EJ, et al. (2005) It Is Time to Implement Routine, Not Risk-Based, HIV Testing. Clin Infect Dis 40: 1037-1040.
This article was originally published in a special issue, Epidemiology and Prevention: HIV handled by Editor(s). Dr. Han-zhu Qian, Vanderbilt University School of Medicine, USA 